\title{
Hybridisation of perovskite nanocrystals with organic molecules for highly efficient liquid scintillators
}

\author{
Sangeun Cho', Sungwoo Kim², Jongmin Kim¹, Yongcheol Jo' ${ }^{1}$ Ilhwan Ryu ${ }^{3}$, Seongsu Hong ${ }^{1}$, Jae-Joon Lee ${ }^{3}$, \\ SeungNam Cha ${ }^{4}$, Eun Bi Nam ${ }^{5}$, Sang Uck Lee $\mathbb{B}^{5}$, Sam Kyu Noh' ${ }^{1}$, Hyungsang Kim', Jungwon Kwak $\mathbb{B}^{2}$ and Hyunsik $\operatorname{Im}^{1}$
}

\begin{abstract}
Compared with solid scintillators, liquid scintillators have limited capability in dosimetry and radiography due to their relatively low light yields. Here, we report a new generation of highly efficient and low-cost liquid scintillators constructed by surface hybridisation of colloidal metal halide perovskite $\mathrm{CsPbA}_{3}(\mathrm{~A}: \mathrm{Cl}, \mathrm{Br}, \mathrm{I}$ ) nanocrystals (NCs) with organic molecules (2,5-diphenyloxazole). The hybrid liquid scintillators, compared to state-of-the-art Csl and $\mathrm{Gd}_{2} \mathrm{O}_{2} \mathrm{~S}$, demonstrate markedly highly competitive radioluminescence quantum yields under $\mathrm{X}$-ray irradiation typically employed in diagnosis and treatment. Experimental and theoretical analyses suggest that the enhanced quantum yield is associated with $X$-ray photon-induced charge transfer from the organic molecules to the NCs. High-resolution X-ray imaging is demonstrated using a hybrid $\mathrm{CsPbBr}_{3} \mathrm{NC}$-based liquid scintillator. The novel X-ray scintillation mechanism in our hybrid scintillators could be extended to enhance the quantum yield of various types of scintillators, enabling low-dose radiation detection in various fields, including fundamental science and imaging.
\end{abstract}

\section{Introduction}

Highly sensitive X-ray detection is becoming increasingly important in areas from everyday life to industry, the military, and scientific research ${ }^{1-4}$. Scintillation materials convert X-ray ${ }^{5}, \gamma$-ray ${ }^{6}$, and particle radiation into visible or ultraviolet (UV) light. Among the various properties of scintillation materials, quantum yield (or light output) is the one most closely associated parameters with both the efficiency and resolution of detectors. Because the quantum yield depends on the nature of the incident particles and photons with varying degrees of energy, a proper scintillation material is chosen according to the type of application. Compared with crystalline or plastic

\footnotetext{
Correspondence: Hyungsang Kim (hskim@dongguk.edu)

Jungwon Kwak (jwkwak0301@gmail.com) or Hyunsik Im (hyunsik7@dongguk.edu) 'Division of Physics and Semiconductor Science, Dongguk University, Seoul 04620, Republic of Korea

2Department of Radiation Oncology, Asan Medical Center, Seoul 05505, Republic of Korea

Full list of author information is available at the end of the article
}

scintillators, liquid scintillators generally have better resistance to damage arising from exposure to intense radiation while providing excellent area/volume scalability $^{7,8}$; consequently, liquid scintillators are used for various purposes, such as in $\beta$-ray spectroscopy, radioactivity measurements, and particle physics ${ }^{9,10}$. However, despite the above advantages, liquid scintillators have relatively low density and low radioluminescence (RL) quantum yield, both of which are crucial in achieving high resolution and contrast in X-ray imaging. As a result, liquid scintillators have rarely been utilised in radiation imaging.

Recently, metal halide perovskite materials, including both bulk crystals of organic inorganic hybrid perovskites and nanocrystals (NCs), have been demonstrated ${ }^{11-14}$ to efficiently convert X-ray photons into charge carriers or visible photons ${ }^{15-19}$. In particular, fully inorganic perovskite NCs have advantages such as highly emissive X-raygenerated excitonic states $^{20}$, ultrafast radiative emission

\section{(c) The Author(s) 2020}

(c) Open Access This article is licensed under a Creative Commons Attribution 4.0 International License, which permits use, sharing, adaptation, distribution and reproduction c. in any medium or format, as long as you give appropriate credit to the original author(s) and the source, provide a link to the Creative Commons license, and indicate if changes were made. The images or other third party material in this article are included in the article's Creative Commons license, unless indicated otherwise in a credit line to the material. If material is not included in the article's Creative Commons license and your intended use is not permitted by statutory regulation or exceeds the permitted use, you will need to obtain permission directly from the copyright holder. To view a copy of this license, visit http://creativecommons.org/licenses/by/4.0/. 
rates $^{21}$, and resistance against high-energy radiation ${ }^{22}$, all of which are essential for highly efficient and durable X-ray scintillators. Moreover, perovskite NCs have high optical sensitivity in response to exposure to X-rays and high X-ray absorption efficiency ${ }^{22,23}$. Perovskite NCs are also commonly uniformly dispersed in nonpolar liquid media for use in liquid scintillators. However, despite their unique properties being superior to those of commercially manufactured scintillators, for example, Tl-doped $\mathrm{CsI}^{24}$ and $\mathrm{Gd}_{2} \mathrm{O}_{2} \mathrm{~S}^{25}$, perovskite NCs still require further improvements in their quantum yield for practical applications. Here, we report an experimental investigation of highly efficient X-ray scintillation and significantly enhanced quantum yields of liquid scintillators consisting of perovskite metal halide $\mathrm{CsPbA}_{3}(\mathrm{~A}: \mathrm{Cl}, \mathrm{Br}, \mathrm{I}) \mathrm{NCs}$ and $\mathrm{C}_{15} \mathrm{H}_{11} \mathrm{NO}$ (2,5-diphenyloxazole: PPO) organic molecules in soft and hard $\mathrm{X}$-ray regimes and demonstrate their use in high-resolution X-ray imaging. We propose a new type of mechanism for substantially enhancing the scintillation quantum yield, which is accomplished by hybridising different scintillation nanomaterials.

\section{Results}

\section{Hybrid $\mathrm{CsPbA}_{3}$ liquid scintillators and radiography}

The hybrid liquid scintillators were manufactured by dispersing $\mathrm{CsPbA}_{3} \mathrm{NCs}$ and PPO in octane without precipitation (Fig. 1a). The perovskite NCs were synthesised via a hot injection method ${ }^{26-28}$ (see "Methods" for details). Transmission electron microscopy (TEM) measurements revealed that the as-synthesized NCs have a cubic shape with an average size of $12 \mathrm{~nm}$ (Fig. 1b). The optical and structural properties of the perovskite NCs were investigated using photoluminescence (PL), ultraviolet-visible (UV-Vis) spectroscopy, X-ray diffraction (XRD) measurements, and TEM images (Supplementary Figs. S1 and S2) ${ }^{29-31}$. To quickly evaluate the suitability of the $\mathrm{CsPbBr}_{3} \mathrm{NCs}+\mathrm{PPO}$ material as a scintillator for X-ray imaging, we imaged a wide range of biological and inorganic specimens with $\mathrm{X}$-rays using a liquid scintillator panel (Fig. 1c) combined with a chargecoupled device (CCD) camera (Fig. 1d). For radiographic measurements, the specially designed display panel was used. The colloidal hybrid $\mathrm{CsPbBr} \mathrm{P}_{3} \mathrm{NCs}+\mathrm{PPO}$ solution was sandwiched by two quartz windows with 4-inch diameters. The X-ray images were taken at an accelerating voltage of $70 \mathrm{kVp}$. To demonstrate $\mathrm{X}$-ray imaging, the concentrations of the $\mathrm{CsPBr}_{3} \mathrm{NCs}$ and $\mathrm{PPO}$ in octane were set at $25 \mathrm{mg} / \mathrm{ml}$ and $10 \mathrm{mg} / \mathrm{ml}$, respectively. An object was placed on the panel detector, and an X-rayexcited optical image was projected through a mirror onto the CCD. As will be discussed in further detail, the $\mathrm{CsPbBr}_{3} \mathrm{NCs}+\mathrm{PPO}$ scintillator was selected to demonstrate the X-ray imaging because the $\mathrm{CsPbBr}_{3} \mathrm{NCs}$ have excellent durability and the strongest RL intensity. As shown in Fig. 1e-g and Supplementary Fig. S3, the metal structures within the biological and plastic specimens were clearly imaged on the liquid scintillator panel.

\section{Enhanced radioluminescence in hybrid $\mathrm{CsPbA}$ scintillators}

Figure $2 \mathrm{a}$ shows photographs of the $\mathrm{X}$-ray imaging system and colloidal $\mathrm{CsPbA}_{3} \mathrm{NCs}+\mathrm{PPO}$ scintillators in the presence of white light and under X-ray irradiation (accelerating voltage: $6 \mathrm{MVp}$ ). During $\mathrm{X}$-ray exposure, the $\mathrm{CsPbBr}{ }_{3} \mathrm{NCs}+\mathrm{PPO}$ scintillator exhibited the brightest RL and emitted a green colour. As anticipated, the hybrid $\mathrm{CsPbBr}{ }_{3} \mathrm{NCs}+\mathrm{PPO}$ scintillator exhibited the highest RL intensity in both the soft and hard X-ray regimes (Supplementary Fig. S4).

Figure $2 \mathrm{~b}$ shows a comparison of the RL spectra emitted from the $\mathrm{CsPbBr}_{3} \mathrm{NCs}(25 \mathrm{mg} / \mathrm{ml}), \mathrm{PPO}(10 \mathrm{mg} / \mathrm{ml})$, and hybrid $\mathrm{CsPbBr}_{3} \mathrm{NCs}+\mathrm{PPO}$ scintillators. The hybrid NCs + PPO scintillator exhibited strong RL that was several times stronger than those emitted by other scintillators. The hybrid NCs + PPO and pure NCs scintillators had the same RL peak positions, indicating that adding PPO does not significantly affect the emission energy of the $\mathrm{CsPbBr}_{3} \mathrm{NCs}$ while enhancing their RL intensity. Another interesting observation is that the RL signal of PPO completely disappears in the spectrum of the hybrid NCs $+\mathrm{PPO}$ scintillator, suggesting the likelihood of X-rayinduced charge transfer from PPO to the $\mathrm{CsPbBr}_{3} \mathrm{NCs}$. We have experimentally demonstrated that the RL spectrum of a powder mixture containing the same amounts of PPO and $\mathrm{CsPbBr}_{3} \mathrm{NCs}$ without octane exhibited two resolved RL emissions, each corresponding to PPO and $\mathrm{CsPbBr}_{3} \mathrm{NCs}$ (Supplementary Fig. S5) and that the PPO peak is not suppressed in the PL spectrum of the hybrid NCs+PPO scintillator under UV irradiation (see Supplementary Fig. S6); collectively, these findings support the proposed mechanism that PPO plays a key role in enhancing the RL of the $\mathrm{CsPbA}_{3} \mathrm{NCs}$ in octane. The surface hybridisation of halide perovskite NCs with $\mathrm{PPO}$ is highly feasible in a nonpolar liquid solvent medium such as octane. The same dramatic RL enhancement was also observed with the hybrid $\mathrm{CsPCl}_{3} \mathrm{NCs}+\mathrm{PPO}$ and $\mathrm{CsPb}_{3} \mathrm{NCs}+\mathrm{PPO}$ scintillators (Fig. 2c).

\section{Scintillation mechanism}

In lead halide perovskite NCs, the photoelectric interaction between incident high-energy X-ray photons and heavy lattice atoms produces high-energy electrons, and these energetic electrons subsequently generate secondary high-energy carriers ${ }^{32,33}$. The hot carriers then undergo a thermalisation process, producing numerous low-energy excitons, and, consequently, high-energy X-ray photons are converted to visible low-energy photons via directbandgap luminescence ${ }^{23}$. For our hybrid $\mathrm{CsPbA}_{3} \mathrm{NCs}$ $+\mathrm{PPO}$ scintillators, $\mathrm{X}$-ray-induced energetic electrons 


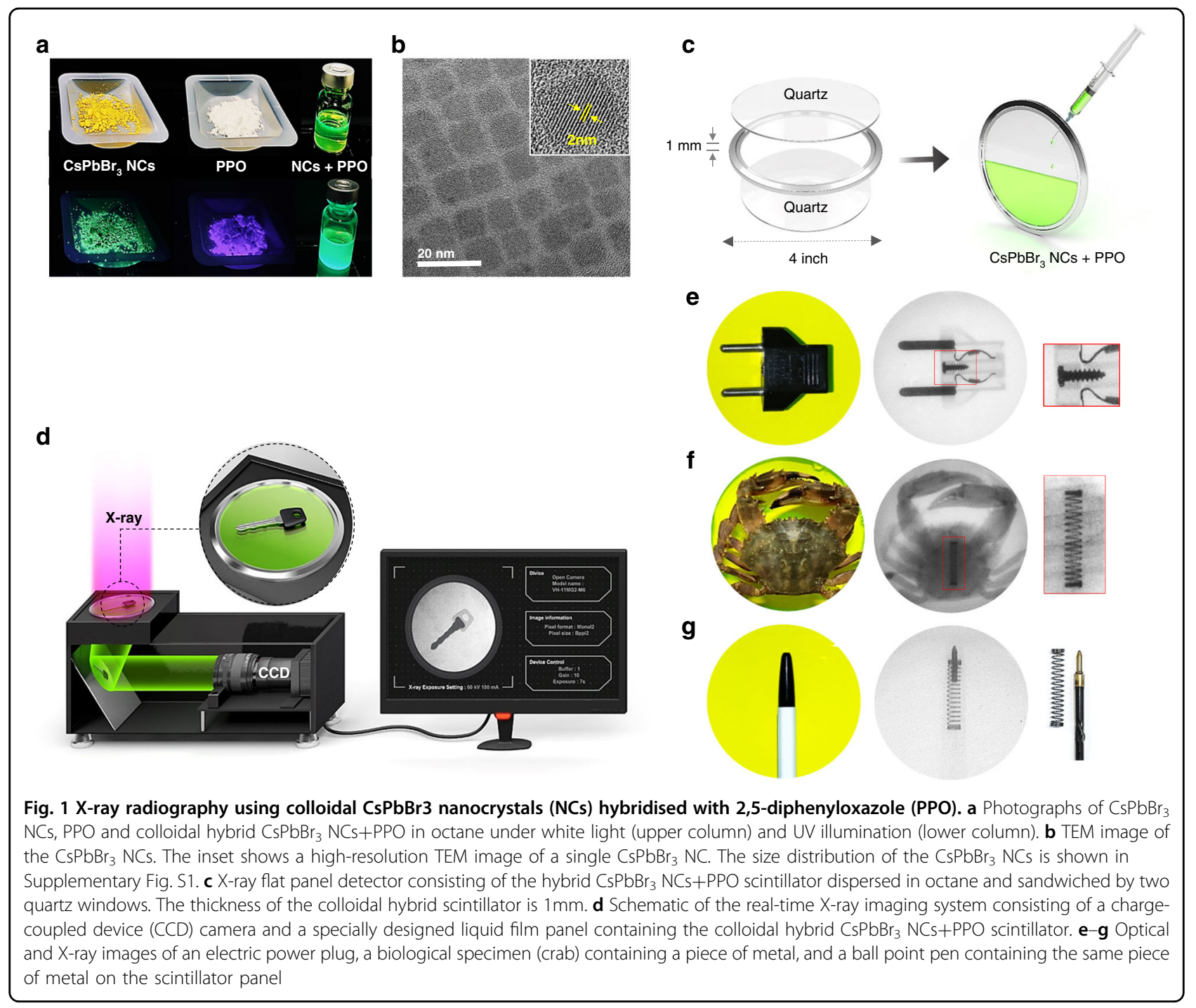

generated from PPO can transfer to the $\mathrm{CsPbA}_{3} \mathrm{NCs}$ via surface hybridisation and amplify the number of energetic electrons in the NCs, thereby enhancing the RL from the $\mathrm{CsPbA}_{3} \mathrm{NCs}$ with a significantly improved quantum yield (Fig. 2d).

Density functional theory (DFT) calculations were performed to simulate the surface hybridisation of $\mathrm{CsPbBr}_{3}$ NCs with PPO and elucidate the origin of the improved quantum yield in the hybrid $\mathrm{CsPbBr}_{3} \mathrm{NCs}+\mathrm{PPO}$ scintillator in terms of X-ray-induced charge transfer from $\mathrm{PPO}$ to the NCs. For hybridisation of the $\mathrm{CsPbBr}_{3} \mathrm{NCs}$ with $\mathrm{PPO}$, the PPO must compete with the oleic acid (OA) ligand bound to the $\mathrm{CsPbBr}_{3} \mathrm{NC}$ surfaces via surface reactions. Thus, we first compared the binding energies of $\mathrm{PPO}$ and $\mathrm{OA}$ on the $\mathrm{CsPbBr}_{3} \mathrm{NC}$ surfaces and assessed how well the desorbed OA could be dissolved in octane.

Neutral PPO and anionic OA showed binding energies of $-1.03 \mathrm{eV}$ and $-0.30 \mathrm{eV}$ on the $\mathrm{Pb}$ site, respectively, and anionic $\mathrm{OA}$ had a larger solvation free energy of $-36.05 \mathrm{kcal} / \mathrm{mol}$ compared with the value of $-9.82 \mathrm{kcal} /$ mol for PPO in octane solvent. The calculated results revealed that $\mathrm{PPO}$, with its relatively large binding energy, can replace the $\mathrm{OA}$ on the $\mathrm{CsPbBr}_{3} \mathrm{NC}$ surface and that the desorbed OA can be stabilised in octane with a large negative solvation free energy (Supplementary Table S1). Therefore, the formation of the hybrid $\mathrm{CsPbBr}_{3} \mathrm{NCs}+\mathrm{PPO}$ in octane was facilitated by the strong interaction between $\mathrm{PPO}$ and $\mathrm{Pb}$ ion sites through $\mathrm{N}-\mathrm{Pb}$ bonding (Supplementary Figs. S7 and S8). XPS measurements of $\mathrm{CsPbBr}_{3} \mathrm{NCs}, \mathrm{PPO}$, and $\mathrm{CsPbBr}_{3} \mathrm{NCs}$ $+\mathrm{PPO}$ provide strong evidence for $\mathrm{N}-\mathrm{Pb}$ bonding (Supplementary Fig. S9).

We analysed the energy level alignment between PPO and the $\mathrm{CsPBBr}_{3} \mathrm{NCs}$ and the frontier orbital distributions. X-ray-induced charge transfer was allowed when the excited state of PPO was much higher than the 

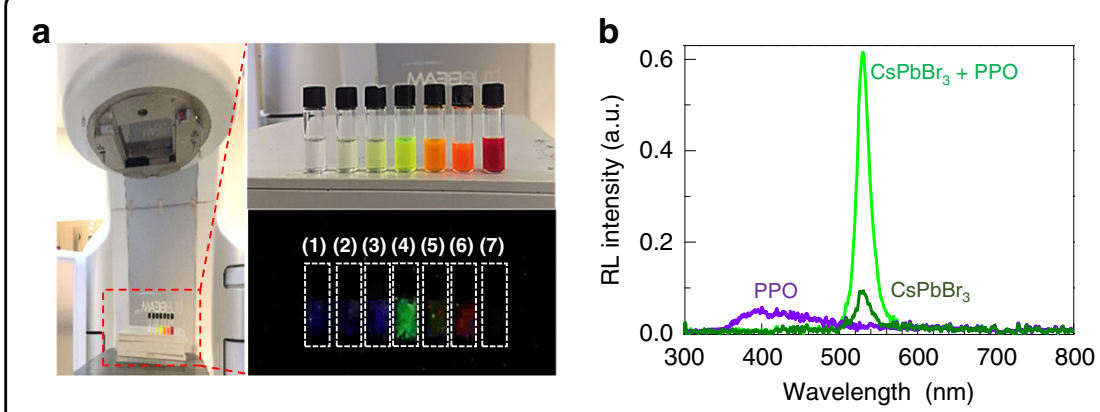

d

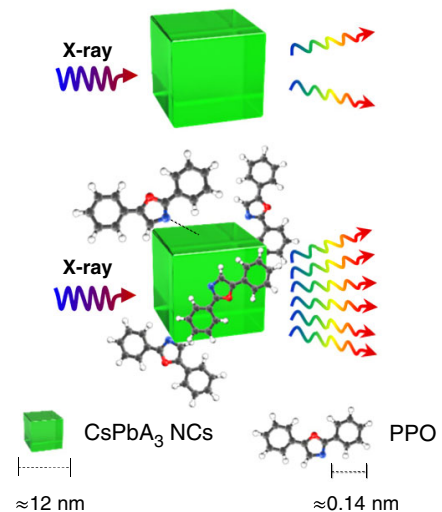

e

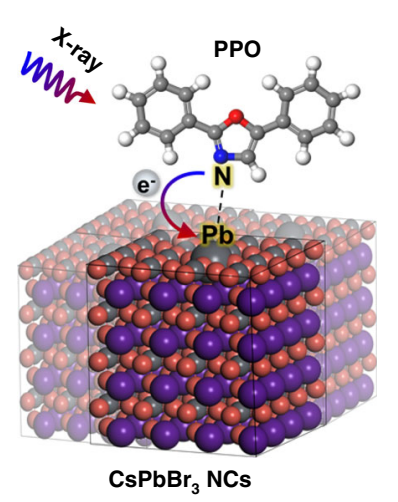

C

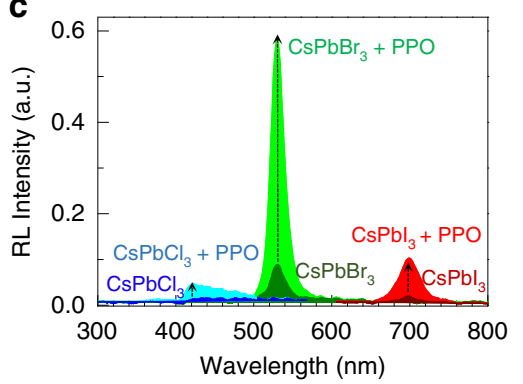

f

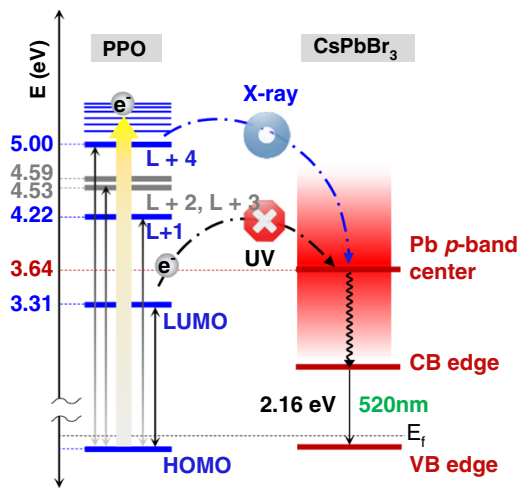

Fig. 2 Enhanced RL of CsPbA3 NCs+PPO (A: Cl, Br, I) hybrid materials in octane. a X-ray generator used for X-ray imaging and RL measurements. The magnified photographs show the hybrid $\mathrm{CsPbA}_{3} \mathrm{NCs}+\mathrm{PPO}$ samples in ambient light and under $\mathrm{X}$-ray irradiation. The material

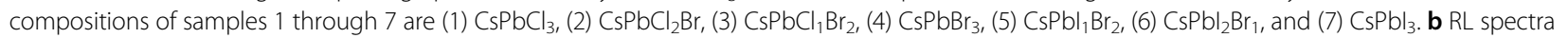
of the hybrid $\mathrm{CsPbBr}_{3} \mathrm{NCs}+\mathrm{PPO}, \mathrm{CsPbBr}_{3} \mathrm{NCs}$, and PPO scintillators. c RL spectra of the hybrid $\mathrm{CsPbA}_{3} \mathrm{NCs}_{2} \mathrm{PPO}$ and CsPbA $\mathrm{NCs}_{3}$ scintillators. d Schematic illustration of the RL of a $\mathrm{CsPbA}_{3} \mathrm{NC}$ and a $\mathrm{CsPbA}_{3} \mathrm{NC}$ hybridised with PPO. e Schematic diagram describing the hybridisation of a $\mathrm{CsPbBr}_{3} \mathrm{NC}$ with PPO. The negatively charged $\mathrm{N}$ in the PPO binds to the positively charged Pb sites on the (001) surface of the CsPbBr $\mathrm{NC}_{3} \mathbf{f} \mathrm{DFT}$ calculations of the energy level alignment for the proposed mechanism of enhanced RL in the hybrid $\mathrm{CsPbBr}_{3} \mathrm{NCs}+\mathrm{PPO}$ scintillator. Under X-ray irradiation, a high-energy electron (e in the solid circle) generated in the PPO moves to $\mathrm{CsPbBr}_{3} \mathrm{NCs}$

conduction band state of the $\mathrm{CsPbBr}_{3} \mathrm{NCs}$. In particular, the large contribution of $\mathrm{N}$ and $\mathrm{Pb}$ in forming $\mathrm{N}-\mathrm{Pb}$ bonds that led to the aligned states of $\mathrm{PPO}$ and the $\mathrm{CsPbr}_{3} \mathrm{NCs}$ effectively led to charge transfer from $\mathrm{PPO}$ to the $\mathrm{CsPbBr}_{3}$ NCs (Fig. 2e and Supplementary Fig. S10). Here, we used the $p$-band centre of the $\mathrm{Pb}$ atom as the representative conduction band state of the $\mathrm{CsPbBr}_{3}$ NCs because the valence $6 p$-orbital of $\mathrm{Pb}$ is involved in the $\mathrm{N}-\mathrm{Pb}$ bond and is distributed over a wide range of conduction bands with various contributions. The energy level alignment in Fig. $2 \mathrm{f}$ revealed that the lowest unoccupied molecular orbital (LUMO) state of PPO and the $p$-band centre of the $\mathrm{Pb}$ atom were located at $3.31 \mathrm{eV}$ and $3.64 \mathrm{eV}$, respectively. Energy levels are denoted based on the aligned Fermi energy $\left(\mathrm{E}_{\mathrm{f}}\right)$ of the hybrid $\mathrm{CsPbBr}{ }_{3} \mathrm{NCs}+\mathrm{PPO}$ at $0 \mathrm{eV}$. In addition, the $\mathrm{N}$ atom in PPO significantly contributed to $\mathrm{LUMO}$, LUMO+1, and LUMO +4 ; therefore, the LUMO +1 and LUMO +4 states, which were located above the $p$-band centre of the $\mathrm{Pb}$ atom and had large contributions from the $\mathrm{N}$ atom, could effectively induce charge transfer from PPO to the $\mathrm{CsPbBr}_{3}$ NCs. This implies that a sufficiently high-energy source, such as X-ray irradiation, is required to induce charge transfer from the excited states above the LUMO of PPO to the $\mathrm{Pb} p$-orbital of the $\mathrm{CsPbBr}_{3} \mathrm{NCs}$.

Consequently, the characteristic structural and electronic features of the hybrid $\mathrm{CsPbBr}_{3} \mathrm{NCs}+\mathrm{PPO}$ scintillator resulted in selective charge transfer under X-ray irradiation, eventually enhancing the scintillation quantum yield. On the other hand, an excited electron in PPO cannot move to an NC upon UV illumination because the energy levels of the allowed states in the NC are too high. This is consistent with the experimental observation that lowenergy UV light cannot enhance the quantum yield in the hybrid NCs+PPO scintillator (Supplementary Fig. S11).

\section{Characterisation of radioluminescence}

We then measured the RL spectra of the $\mathrm{CsPbA}_{3} \mathrm{NC}$ $(25 \mathrm{mg} / \mathrm{ml})+\mathrm{PPO}(10 \mathrm{mg} / \mathrm{ml})$ hybrid scintillators as a function of dose rate (Supplementary Fig. S12). The 


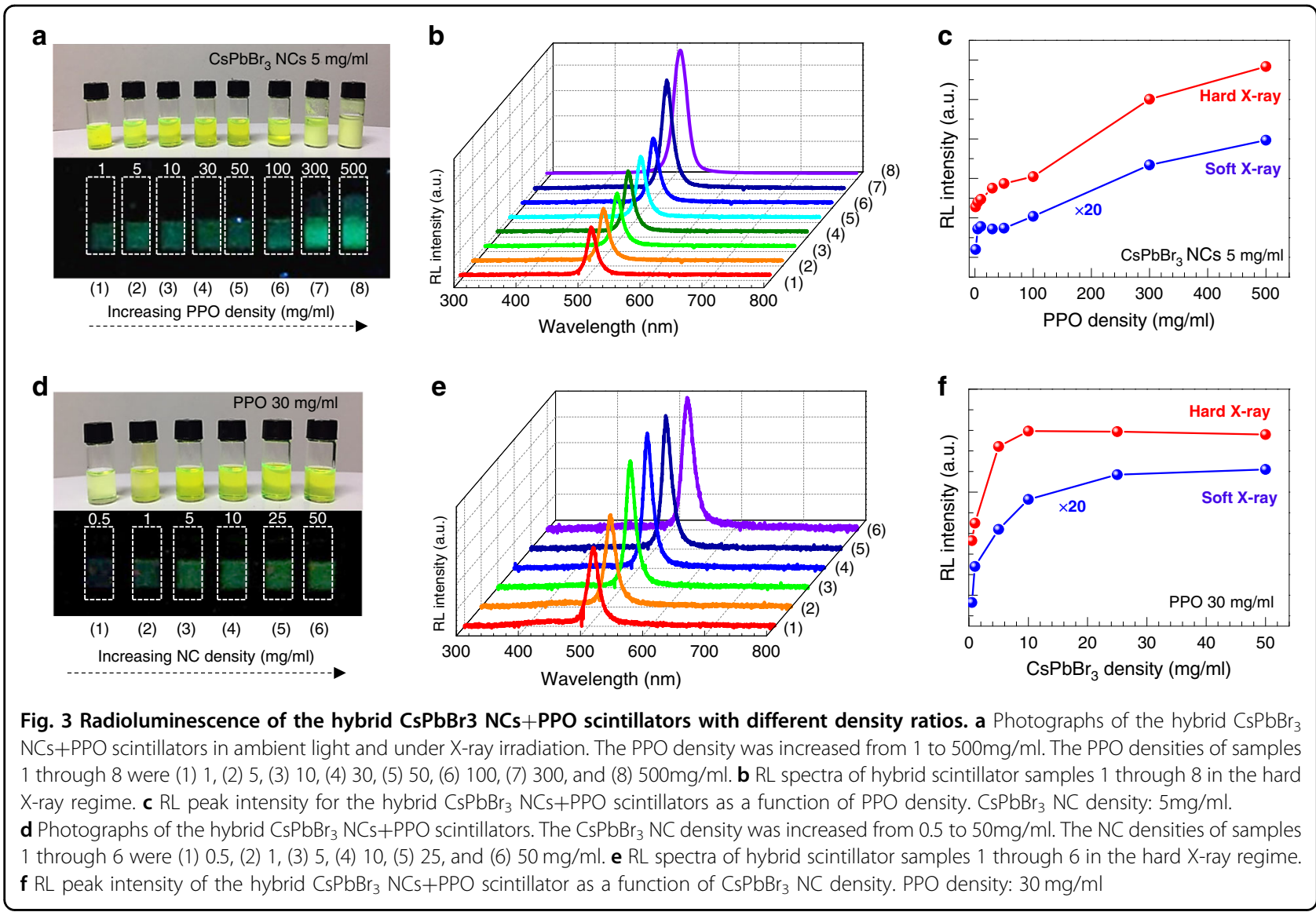

measured RL emission exhibited a linear response to the $\mathrm{X}$-ray dose rate, which is a desirable feature of a good scintillator for X-ray imaging and dosimetry. We also measured the X-ray response characteristics of the hybrid scintillator upon excitation with a single X-ray photon from a portable coin-type ${ }^{60} \mathrm{Co}$ source (Supplementary Fig. S13). The extracted fast scintillation decay time $\tau$ was 60-100 ns for the $\mathrm{CsPbA}_{3} \mathrm{NCs}+\mathrm{PPO}$ hybrid scintillators, which is much shorter than that for the bulk CsI:Tl (on the order of $\mu \mathrm{s}$ ). The fast RL decay time of the hybrid scintillators is also expected to act as a favourable trait for use in medical radiography.

We further quantitatively investigated how PPO contributes to the RL of the $\mathrm{CsPbBr}_{3} \mathrm{NCs}+\mathrm{PPO}$ hybrid scintillator by varying the concentration ratio of the $\mathrm{NCs}$ and PPO. As shown in the photographs (Fig. 3a) under Xray irradiation (accelerating voltage: $6 \mathrm{MV}_{\mathrm{p}}$ ), the $\mathrm{RL}$ emission from the hybrid $\mathrm{NCs}+\mathrm{PPO}$ scintillator became brighter as the PPO density was increased for a fixed NC density of $5 \mathrm{mg} / \mathrm{ml}$. We measured the RL spectra of the hybrid NCs+PPO scintillators in the soft (dose rate of $37.4 \mathrm{mGy} \mathrm{s}^{-1}$ at an accelerating voltage of $50 \mathrm{kVp}$ ) and hard X-ray regimes (Fig. 3b, Supplementary Fig. S14a), plotted the measured RL peak intensity as a function of the PPO density (Fig. 3c), and observed a linear relationship between the RL intensity and PPO density. The scintillation efficiency of a hybrid $\mathrm{CsPbBr}_{3}+\mathrm{PPO}$ scintillator was enhanced with increasing PPO density (Supplementary Fig. S15). Without $\mathrm{CsPbBr}_{3} \mathrm{NCs}$, the RL intensity of the pure PPO liquid scintillator decreased at high PPO densities $(>10 \mathrm{mg} / \mathrm{ml})$, which was likely due to scintillation quenching (namely, self-absorption) ${ }^{34,35}$ (Supplementary Fig. S16). When the PPO density was greater than $50 \mathrm{mg} / \mathrm{ml}$ in the colloidal hybrid scintillator, a yellowish-green dense precipitate was formed. As the PPO density was further increased to above the critical value of $\sim 500 \mathrm{mg} / \mathrm{ml}$, the hybrid $\mathrm{NCs}+\mathrm{PPO}$ material in octane completely transformed into an opaque dense precipitate that emitted a very strong RL.

We also carried out similar measurements while increasing the $\mathrm{CsPbBr}{ }_{3} \mathrm{NC}$ density for a fixed PPO density of $30 \mathrm{mg} / \mathrm{ml}$ and observed that precipitates were not formed. In contrast, as the $\mathrm{CsPbBr}_{3} \mathrm{NC}$ density increased, the RL emission quickly saturated in both the soft and hard X-ray regions, as shown in the photographs (Fig. 3d) and RL spectra (Fig. 3e and Supplementary Fig. S14b). The observed features are summarised in Fig. 3f, in which the measured RL peak intensity is plotted as a function of NC density. 


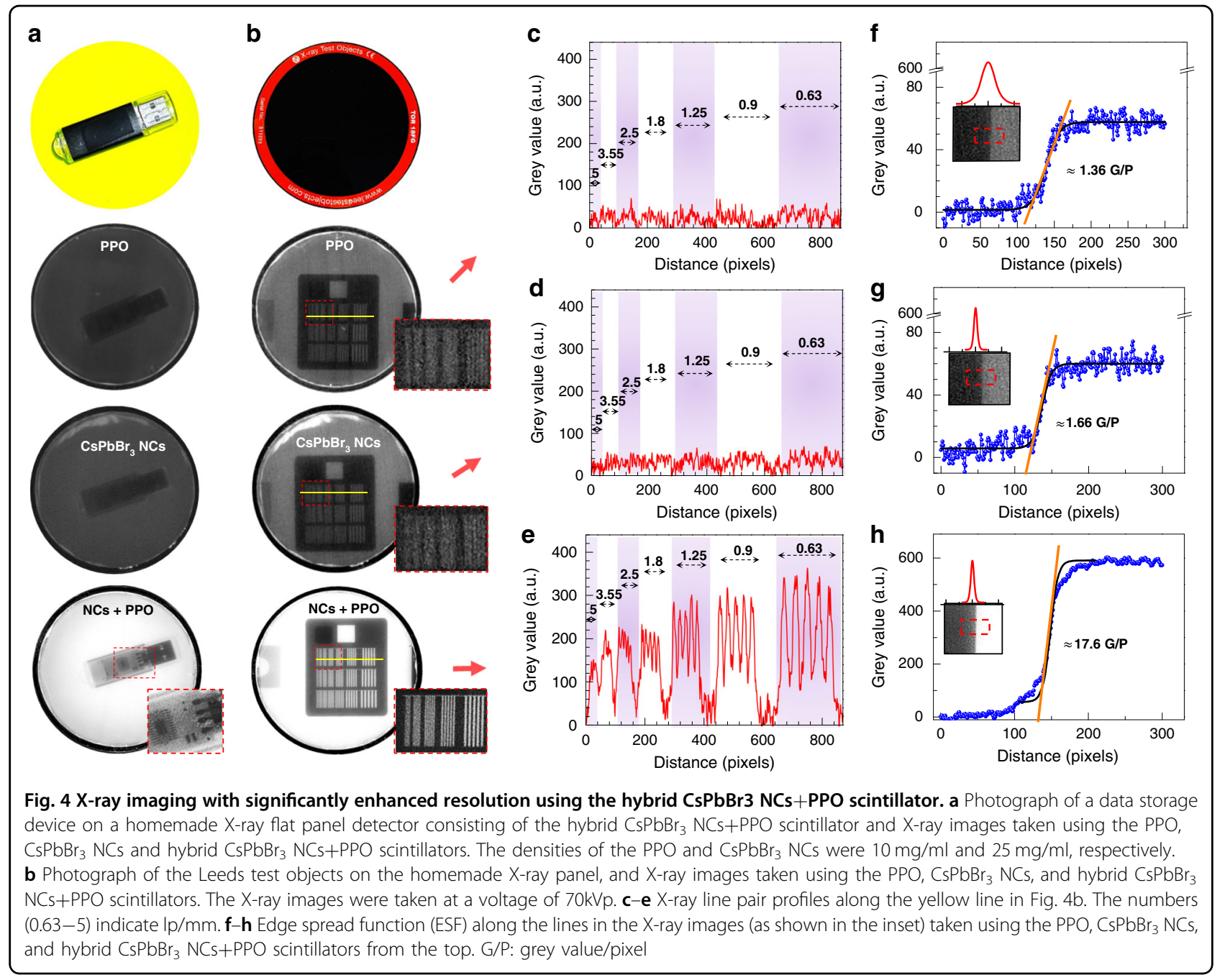

\section{X-ray imaging performance}

To directly confirm any enhancement in the $\mathrm{X}$-ray image quality when using the hybrid $\mathrm{CsPbBr}{ }_{3} \mathrm{NCs}+\mathrm{PPO}$ liquid scintillator, we recorded the $\mathrm{X}$-ray images of a portable data storage device using $\mathrm{PPO}, \mathrm{CsPbBr}{ }_{3} \mathrm{NC}$, and hybrid $\mathrm{CsPbBr}_{3} \mathrm{NCs}+\mathrm{PPO}$ scintillators (Fig. 4a). The hybrid $\mathrm{NCs}+\mathrm{PPO}$ scintillator produced notably clearer $\mathrm{X}$-ray images than those of PPO the $\mathrm{CsPBrr}_{3}$ NCs. The spatial resolution and image quality of the scintillation materials were quantitatively evaluated using a radiography test phantom ${ }^{36,37}$ (Leeds test objects, model: TOR 18FG, Supplementary Fig. S17). Figure 4b shows X-ray images of the test objects. The imaging performances of the scintillators were comparatively evaluated by counting the maximal number of resolvable line pairs per millimetre $(\mathrm{lp} / \mathrm{mm})$ and checking the abruptness of the contrast changes at the boundary. Figure $4 \mathrm{c}-\mathrm{e}$ shows the intensity variation along the yellow lines in the X-ray images of the line patterns. The largest detectable $\mathrm{lp} / \mathrm{mm}$ of the hybrid scintillator was at least $3.5 \mathrm{lp} / \mathrm{mm}$, which was several times greater than those of the pure halide perovskite NCs and PPO scintillators.

Figure $4 \mathrm{f}-\mathrm{h}$ show the edge spread functions (ESFs) at the boundary of the test phantom, indicated by the red dashed boxes in the insets, which characterise the sharpness of the images. The abrupt change in intensity is reflected by the slope across the boundary. The measured slope was 17.6 grey value/pixel for the hybrid $\mathrm{NCs}+\mathrm{PPO}$ scintillator, which was much larger than those for the other materials (range, 1.36-1.66 grey value/pixel) (pixel size: $9 \mu \mathrm{m}$ ). The line spread functions (LSFs) extracted from the ESFs (red curves above the insets) also indicated how sharp the image was near the boundary in terms of the full width at half maximum (FWHM $)^{38}$. The estimated FWHM of the NCs+PPO hybrid material was 7.6 pixels, which is much smaller than those of the other scintillators (range: 16-32 pixels). The image contrast is a measure of how clearly an object is distinguishable and can be 
assessed using the following expression:

$$
\text { Contrast }(\%)=100 \times\left(I_{\text {Object }}-I_{\text {Background }}\right) /\left(I_{\text {Object }}+I_{\text {Background }}\right)
$$

where $I_{\text {Object }}$ and $I_{\text {Background }}$ represent the RL intensities of the object and adjacent material near the boundary, respectively. The contrast near the boundary was 33\% for the hybrid NCs+PPO scintillator and $12-13 \%$ for the other scintillators.

Ageing and deterioration of the hybrid $\mathrm{NCs}+\mathrm{PPO}$ scintillator were examined by repeating the X-ray imaging measurements in the same environment after a year (Supplementary Fig. S18) and after continuous irradiation with a very high-energy X-ray for a prolonged period (Supplementary Fig. S19). The colloidal hybrid scintillator exhibited almost no degradation in performance, thereby confirming its stability.

\section{Discussion}

In conclusion, we developed a new type of liquid scintillator by hybridising colloidal halide perovskite $\mathrm{CsPA}_{3}$ (A: $\mathrm{Cl}, \mathrm{Br}, \mathrm{I}$ ) nanocrystals with 2,5-diphenyloxazole (PPO) and demonstrated that the novel liquid scintillator has a very high quantum yield that allows for efficient X-ray detection. Considering their additional advantages, including cost-effective mass production, stability under high-energy X-ray irradiation, and easy processability in combination with various substances, these novel hybrid nanomaterials are suitable as scintillators for a wide range of X-ray technologies that require high-performance detectors and imagers. While the fundamentals of scintillation in these halide perovskite NCs+PPO hybrid nanomaterials require further elucidation, these colloidal hybrid nanocrystals hold substantial promise for advancing the industrial applications of X-ray imaging and producing intriguing scintillation in hybrid nanomaterials.

\section{Materials and methods \\ Chemicals}

Caesium carbonate $\left(\mathrm{Cs}_{2} \mathrm{CO}_{3}, 99.9 \%\right)$, lead iodide $\left(\mathrm{PbI}_{2}\right.$, 99.9\%), lead bromide $\left(\mathrm{PbBr}_{2}, 99.9 \%\right)$, lead chloride $\left(\mathrm{PbCl}_{2}\right.$, 99.9\%), oleic acid (OA, technical grade, $90 \%)$, oleylamine (OAm, technical grade 70\%), 1-octadecene (ODE, technical grade $90 \%)$, n-octane (99\%, Germany), and 2,5-diphenyloxazole (PPO, 99\%) were purchased from Sigma-Aldrich.

\section{Preparation of Cs-oleate}

The Cs-oleate precursor was synthesised using the conventional hot injection method ${ }^{26-28} \cdot \mathrm{Cs}_{2} \mathrm{CO}_{3}(0.407 \mathrm{~g})$, OA $(1.25 \mathrm{ml})$ and $\mathrm{ODE}(15 \mathrm{ml})$ were dissolved in a 3 necked round-bottom flask by heating under vacuum at $120^{\circ} \mathrm{C}$ for $60 \mathrm{~min}$ with magnetic stirring. To ensure a complete reaction between $\mathrm{Cs}_{2} \mathrm{CO}_{3}$ and $\mathrm{OA}$, the mixture was heated at $150{ }^{\circ} \mathrm{C}$ for $60 \mathrm{~min}$ in $\mathrm{N}_{2}$.

\section{Synthesis of $\mathrm{CsPbA}_{3}(\mathrm{~A}: \mathrm{Cl}, \mathrm{Br}, \mathrm{I})$ nanocrystals and hybrid scintillators \\ The $\mathrm{CsPbA}_{3}$ nanocrystals were prepared using the} conventional hot injection method. ODE $(25 \mathrm{ml})$ and $1.89 \mathrm{mmol}$ lead halide $\left(\mathrm{PbI}_{2}: 0.436 \mathrm{~g}\right)$, lead bromide $\left(\mathrm{PbBr}_{2}: 0.347 \mathrm{~g}\right)$, or lead chloride $\left(\mathrm{PbCl}_{2}: 0.263 \mathrm{~g}\right)$ were dissolved in a 3-necked round bottom flask by heating the mixture at $120^{\circ} \mathrm{C}$ for $60 \mathrm{~min}$ with magnetic stirring under vacuum. Then, the reaction temperature was adjusted from $150-180^{\circ} \mathrm{C}$ depending on the lead halide source. Then, the dried OA $(2.5 \mathrm{ml})$ and OAm $(2.5 \mathrm{ml})$ were injected under $\mathrm{N}_{2}$. After $30 \mathrm{~min}, 2 \mathrm{ml}$ of the as-prepared Cs-oleate solution was quickly injected into the reaction mixture solution. As soon as the solution exhibited various colours, corresponding to the perovskite $\left(\mathrm{CsPbA}_{3}\right)$ $\mathrm{NCs}$ for each lead halide $\left(\mathrm{PbA}_{2}\right)$, the solution was cooled down in an ice-water bath. The synthesized $\mathrm{CsPbA}_{3} \mathrm{NC}$ powder was purified by adding hexane and methyl acetate (volume ratio of 1:1) and centrifuged at $8500 \mathrm{rpm}$. The precipitated $\mathrm{CsPbA}_{3} \mathrm{NCs}$ were redispersed in octane containing PPO.

\section{Density functional theory calculations}

All ab initio calculations were performed with the

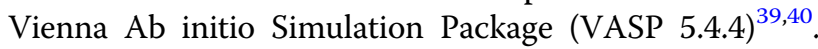
We used the Perdew-Burke-Ernzerhof (PBE) exchangecorrelation functional and the projector augmented-wave (PAW) method ${ }^{41}$. Calculations for geometric optimisation were carried out in a periodically repeated surface $(3 \times 3)$ supercell with $1 \times 1 k$-point sampling. A four-layered slab model was employed for $\mathrm{CsPbBr}_{3}$ (001), separated by a $15 \AA$ vacuum space in the $z$-direction to avoid interaction between layers. In addition, the two topmost layers were allowed to fully relax, while the other layers were fixed to their optimised bulk positions. A plane-wave cut-off energy of $500 \mathrm{eV}$ was used. Lattice constants and internal atomic positions were fully optimised until the residual forces were $<0.04 \mathrm{eV} / \AA$. The schematics of the models are shown in Figs. S5 and S6. To investigate the electronic structures, we employed the Heyd-Scuseria-Ernzerhof (HSE06) hybrid functional calculation ${ }^{42}$ using the GGAPBE-optimised structures. The solvation free energy $\left({ }_{\text {solv }}^{\Delta \mathrm{G}}\right)$ calculations were performed using the solvation model based on density (SMD) ${ }^{43}$ at the B3LYP/6-311 + G (2d,p) level of theory with the Gaussian09 package ${ }^{44}$.

\section{Radioluminescence and X-ray imaging}

To evaluate the feasibility of the fabricated scintillation materials in radiation imaging applications, the scintillation characteristics were confirmed under diagnostic X-ray irradiation. The radioluminescence (RL) was measured in 
the diagnostic energy region of X-rays by varying the tube voltages $(10-300 \mathrm{kVp})$ and currents $(5-40 \mathrm{~mA})$ using an $\mathrm{X}$-ray irradiator (X-RAD 320TM, Precision, USA). The amount of radiation absorbed by the scintillation materials was verified by using radiochromic films (Ashland, USA) widely used for radiation dose measurements.

In the therapeutic energy region of X-rays, the RL was also measured using medical linear accelerators for cancer treatment. The RL spectra of the scintillation materials were measured at room temperature under X-ray irradiation. The spectra were recorded using a compact spectrometer that could measure a wavelength range of 200-1000 nm (CCS200, Thorlabs). The scintillation light was transmitted through a $\varnothing 200 \mu \mathrm{m}$ core fibre optically coupled to the spectrometer.

Radiography was performed using a specially designed panel containing the scintillation material ( $\mathrm{PPO}, \mathrm{CsPbA}_{3}$ $\mathrm{NCs}$ or $\mathrm{PPO}+\mathrm{CsPbA}_{3} \mathrm{NCs}$ ) dissolved in octane. The diameter and thickness of the panel were 4 inches and $1 \mathrm{~mm}$, respectively. The X-ray imaging of the fabricated scintillator was examined for $\mathrm{kVp}$ and MVp X-ray irradiation using a medical LINAC (VitalBeam ${ }^{\circledR}$, Varian, USA) equipped with an On-Board Imager ${ }^{\circledR} \mathrm{kV}$ imaging system.

\section{Radiation decay time}

The measurement system consisted of two photomultiplier tubes (PMTs) that sensed the scintillation light emitted from the scintillator material. As shown in the figure, PMMA discs with $1 \mathrm{ml}$ sample vials were optically bonded between the two facing PMTs and irradiated with ${ }^{60}$ Co gamma-rays. The signals from the two PMTs were acquired with a $500 \mathrm{MHz}$ FADC to measure the amount and decay time of the scintillation light of all individual events. The event triggering condition was set to be the coincidence of the two channels.

\section{Acknowledgements}

This work is supported by the National Research Foundation (NRF) of Korea (grants 2018R1A2B6007436, 2018R1A2B6006320, 2019R1I1A1A01048852 and 2016R1A6A1A03012877) and The Korean Association for Radiation Application.

\section{Author details}

${ }^{1}$ Division of Physics and Semiconductor Science, Dongguk University, Seoul 04620, Republic of Korea. ${ }^{2}$ Department of Radiation Oncology, Asan Medical Center, Seoul 05505, Republic of Korea. ${ }^{3}$ Department of Energy and Materials Engineering, Dongguk University, Seoul 04620, Republic of Korea. ${ }^{4}$ Department of Physics, Sungkyunkwan University, Suwon 2066, Republic of Korea. ${ }^{5}$ Department of Bionano Technology and Department of Applied Chemistry, Hanyang University, Ansan 15588, Republic of Korea

\section{Author contributions}

S.C. and H.I. planned the experiment, designed the samples, and performed measurements and data analysis. S.K. and J.K. carried out X-ray measurements. I.R., S.H. and J.-J.L. synthesized the perovskite nanocrystals. J.K., Y. J, S.N.C. and H.K. contributed to the data analysis. E.B.N. and S.U.L. provided theoretical support for the work. S.K.N. participated in experimental discussions and discussed the data. H. Im. wrote the manuscript with feedback from all the authors, with significant contributions from S.C.
Conflict of interest

The authors declare that they have no conflict of interest.

Supplementary information is available for this paper at https://doi.org/ 10.1038/s41377-020-00391-8.

Received: 18 April 2020 Revised: 18 August 2020 Accepted: 20 August 2020 Published online: 07 September 2020

\section{References}

1. Wei, W. et al. Monolithic integration of hybrid perovskite single crystals with heterogenous substrate for highly sensitive X-ray imaging. Nat. Photonics $\mathbf{1 1}$, 315-321 (2017).

2. Liu, J. Y. et al. Flexible, printable soft-X-ray detectors based on all-inorganic perovskite quantum dots. Adv. Mater. 31, 1901644 (2019).

3. Yakunin, S. et al. Detection of X-ray photons by solution-processed lead halide perovskites. Nat. Photonics 9, 444-449 (2015).

4. Wei, H. T. et al. Dopant compensation in alloyed $\mathrm{CH}_{3} \mathrm{NH}_{3} \mathrm{PbBr}_{3-x} \mathrm{Cl}$. perovskite single crystals for gamma-ray spectroscopy. Nat. Mater. 16, 826-833 (2017).

5. Shrestha, S. et al. High-performance direct conversion X-ray detectors based on sintered hybrid lead triiodide perovskite wafers. Nat. Photonics 11, 436-440 (2017).

6. Yakunin, S. et al. Detection of gamma photons using solution-grown single crystals of hybrid lead halide perovskites. Nat. Photonics 10, 585-589 (2016).

7. Zheng, Z. L. et al. Preparation and performance study of a novel liquid scintillator with mixed solvent as the matrix. Nucl. Instrum. Methods Phys. Res. Sect. A: Accelerators, Spectrometers, Detect. Associated Equip. 850, 12-17 (2017).

8. Winslow, L. \& Simpson, R. Characterizing quantum-dot-doped liquid scintillator for applications to neutrino detectors. J. Instrum. 7, P07010 (2012).

9. Bungau, A. et al. Proposal for an electron antineutrino disappearance search using high-rate ${ }^{8}$ Li production and decay. Phys. Rev. Lett. 109, 141802 (2012).

10. Marchi, T. et al. Optical properties and pulse shape discrimination in siloxanebased scintillation detectors. Sci. Rep. 9, 9154 (2019).

11. Cao, F. et al. Shining emitter in a stable host: design of halide perovskite scintillators for X-ray imaging from commercial concept. ACS Nano $\mathbf{1 4}$ 5183-5193 (2020)

12. Wang, C. Y. et al. X-ray excited $\mathrm{CsPb}(\mathrm{Cl}, \mathrm{Br})_{3}$ perovskite quantum dots-glass composite with long-lifetime. J. Eur. Ceram. Soc. 40, 2234-2238 (2020).

13. $\mathrm{Xu}, \mathrm{Q}$. et al. A solution-processed zero-dimensional all-inorganic perovskite scintillator for high resolution gamma-ray spectroscopy detection. Nanoscale 12, 9727-9732 (2020).

14. Zhu, W. J. et al. Low-dose real-time X-ray imaging with nontoxic double perovskite scintillators. Light: Sci. Appl. 9, 112 (2020).

15. Kim, Y. C. et al. Printable organometallic perovskite enables large-area, lowdose X-ray imaging. Nature 550, 87-91 (2017).

16. Wei, H. T. et al. Sensitive $X$-ray detectors made of methylammonium lead tribromide perovskite single crystals. Nat. Photonics 10, 333-339 (2016).

17. Thirimanne, H. M. et al. High sensitivity organic inorganic hybrid X-ray detectors with direct transduction and broadband response. Nat. Commun. $\mathbf{9}$, 2926 (2018).

18. Wei, H. T. \& Huang, J. S. Halide lead perovskites for ionizing radiation detection. Nat. Commun. 10, 1066 (2019).

19. Zhang, Y. H. et al. Metal Halide perovskite nanosheet for $X$-ray high-resolution scintillation imaging screens. ACS Nano 13, 2520-2525 (2019).

20. Becker, M. A. et al. Bright triplet excitons in caesium lead halide perovskites. Nature 553, 189-193 (2018).

21. Hu, F. R. et al. Superior optical properties of perovskite nanocrystals as single photon emitters. ACS Nano 9, 12410-12416 (2015).

22. Heo, J. H. et al. High-performance next-generation perovskite nanocrystal scintillator for nondestructive X-ray imaging. Adv. Mater. 30, e1801743 (2018).

23. Chen, Q. S. et al. All-inorganic perovskite nanocrystal scintillators. Nature $\mathbf{5 6 1}$, 88-93 (2018).

24. Yang, P. et al. Effect of humidity on scintillation performance in $\mathrm{Na}$ and $\mathrm{Tl}$ activated CsI Crystals. IEEE Trans. Nucl. Sci. 61, 1024-1031 (2104).

25. Yasuda, R., Katagiri, M. \& Matsubayashi, M. Influence of powder particle size and scintillator layer thickness on the performance of $\mathrm{Gd}_{2} \mathrm{O}_{2} \mathrm{~S}: \mathrm{Tb}$ scintillators for neutron imaging. Nucl. Instrum. Methods Phys. Res. Sect. A: Accelerators, Spectrometers, Detect. Associated Equip. 680, 139-144 (2012). 
26. Shekhirev, M. et al. Synthesis of cesium lead halide perovskite quantum dots. J. Chem. Educ. 94, 1150-1156 (2017).

27. Wang, D. et al. Polarized emission from $\mathrm{CsPbX}_{3}$ perovskite quantum dots. Nanoscale 8, 11565-11570 (2016).

28. Swarnkar, A. et al. Quantum dot-induced phase stabilization of a-CsPbl 3 perovskite for high-efficiency photovoltaics. Science 354, 92-95 (2016).

29. Baranov, D. et al. Investigation into the photoluminescence red shift in cesium lead bromide nanocrystal superlattices. J. Phys. Chem. Lett. 10, 655-660 (2019).

30. Ai, B. et al. Low temperature photoluminescence properties of $\mathrm{CsPbBr}_{3}$ quantum dots embedded in glasses. Phys. Chem. Chem. Phys. 19, 17349-17355 (2017)

31. Zhang, Y. W. et al. Multicolored mixed-organic-cation perovskite quantum dots (FAxMA1 $-\mathrm{XPbX} 3, \mathrm{X}=\mathrm{Br}$ and I) for white light-emitting diodes. Ind. Eng. Chem. Res. 56, 10053-10059 (2017).

32. Stoumpos, C. C. et al. Crystal growth of the perovskite semiconductor CsPbBr3: a new material for high-energy radiation detection. Cryst. Growth Des. 13, 2722-2727 (2013)

33. Rodnyi, P. A. Physical Processes in Inorganic Scintillators. (CRC Press, Boca Raton, 1997).

34. Yan, D. P. et al. Two-component molecular materials of 2,5-diphenyloxazole exhibiting tunable ultraviolet/blue polarized emission, pump-enhanced luminescence, and mechanochromic response. Adv. Funct. Mater. 24, 587-594 (2014).

35. Chakraborty, S., Harris, K. \& Huang, M. B. Photoluminescence properties of polystyrene-hosted fluorophore thin films. AIP Adv. 6, 125113 (2016).
36. Balter, S. et al. A pilot study exploring the possibility of establishing guidance levels in X-ray directed interventional procedures. Med. Phys. 35, 673-680 (2008).

37. Pogson, E. M. et al. Comparing and evaluating the efficacy of the TOR18FG leeds test X-ray phantom for T-rays. Quant. Imaging Med. Surg. 3, 18-27 (2013).

38. Boone, J. M. \& Seibert, J. A. An analytical edge spread function model for computer fitting and subsequent calculation of the LSF and MTF. Med. Phys. 21, 1541-1545 (1994)

39. Kresse, G. \& Furthmuller, J. Efficient iterative schemes for ab initio totalenergy calculations using a plane-wave basis set. Phys. Rev. B 54, 11169-11186 (1996).

40. Kresse, G. \& Furthmüller, J. Efficiency of ab-initio total energy calculations for metals and semiconductors using a plane-wave basis set. Computational Mater. Sci. 6, 15-50 (1996).

41. Blöchl, P. E. Projector augmented-wave method. Phys. Rev. B 50, 17953-17979 (1994).

42. Heyd, J., Scuseria, G. E. \& Ernzerhof, M. Hybrid functionals based on a screened coulomb potential. J. Chem. Phys. 118, 8207-8215 (2003).

43. Marenich, A. V., Cramer, C. J. \& Truhlar, D. G. Universal solvation model based on solute electron density and on a continuum model of the solvent defined by the bulk dielectric constant and atomic surface tensions. J. Phys. Chem. B 113, 6378-6396 (2009).

44. Frisch, M. J. et al. Gaussian 09, Revision E.01. (Gaussian, Inc., Wallingford CT, 2013). 